Pacific Journal of Mathematics

KLOOSTERMAN INTEGRALS FOR SKEW SYMMETRIC AMEs RALLIS 


\title{
KLOOSTERMAN INTEGRALS FOR SKEW SYMMETRIC MATRICES
}

\author{
Hervé Jacquet and Stephen Rallis
}

\begin{abstract}
If $G$ is a reductive group quasi-split over a number field $F$ and $K$ the kernel of the trace formula, one can integrate $K$ in the two variables against a generic character of a maximal unipotent subgroup $N$ to obtain the Kuznietsov trace formula. If $H$ is the fixator of an involution of $G$, one can also integrate $K$ in one variable over $H$ and in the other variable against a generic character of $N$ : one obtains then a "relative" version of the Kuznietsov trace formula. We propose as a conjecture that the relative Kuznietsov trace formula can be "matched" with the Kuznietsov trace formula for another group $G^{\prime}$. A consequence of this formula would be the characterization of the automorphic representations of $G$ which admit an element whose integral over $H$ is non-zero: they should be functorial image of representations of $G^{\prime}$. In this article, we study the case where $H$ is the symplectic group inside the linear group; we prove the "fundamental lemma" for the situation at hand and outline the identity of the trace formulas. This case is elementary and should serve as a model for the general case.
\end{abstract}

1. Introduction. Let $F$ be a local field. We will denote by $G^{\prime}$ the group $\mathrm{GL}(m)$, regarded as an algebraic group over $F$, by $N^{\prime}$ the group of upper triangular matrices with unit diagonal, by $A^{\prime}$ the group of diagonal matrices and by $W^{\prime}$ the Weyl group of $A^{\prime}$ identified to the group of permutation matrices. Let $\psi$ be a non-trivial additive character of $F$ and $\theta^{\prime}$ the character of $N^{\prime}$ defined by:

$$
\theta^{\prime}(n)=\psi\left(\sum_{1 \leq i \leq m-1} n_{i, i+1}\right) \text {. }
$$

The group $N^{\prime}$ operates on $G^{\prime}$ by

$$
g^{\prime} \mapsto^{t} n_{1}^{\prime} g^{\prime} n_{2}^{\prime}
$$

We say that the orbit of $g^{\prime}$ under $N^{\prime} \times N^{\prime}$ on $G^{\prime}$ is relevant (with respect to $\theta^{\prime}$ ) if $\theta^{\prime} \otimes \theta^{\prime}$ is trivial on the fixator of $g^{\prime}$ in $N^{\prime} \times N^{\prime}$. If $f^{\prime}$ is a smooth function of compact support of $G^{\prime}$, we define for every relevant element $g^{\prime}$ an orbital integral:

$$
I\left(g^{\prime}, f^{\prime}\right)=\int f^{\prime}\left({ }^{t} n_{1}^{\prime} g^{\prime} n_{2}^{\prime}\right) \theta^{\prime}\left(n_{1}^{\prime} n_{2}^{\prime}\right) d n_{1}^{\prime} d n_{2}^{\prime} \text {. }
$$


The integral is taken over the quotient of $N^{\prime} \times N^{\prime}$ by the fixator of $g^{\prime}$. At the moment, we do not specify the choice of the Haar measures. This type of integral is a "Kloosterman integral," the local analogue of a Kloosterman sum (see [G] and the references therein).

Now let $S$ or $S_{F}$ be the space of skew symmetric matrices of size $2 m \times 2 m$ and rank $2 m$. The group $G=\mathrm{GL}(2 m, F)$ operates on $S$ by:

$$
s \mapsto^{t} g s g .
$$

We denote by $N$ the group of upper triangular matrices with unit diagonal, by $A$ the group of diagonal matrices and by $W$ the group of permutation matrices in $G$. In particular, the group $N$ operates on $S$ and its orbits are easily described: each orbit has exactly one representative in the normalizer of $A$. More precisely, every orbit has a representative of the form $w a$ where $w \in W, a \in A, w^{2}=1$ and $w a w=-a$. We let $\theta$ be the character of $N$ defined by:

$$
\theta(n)=\theta^{\prime}\left(n_{1}\right) \theta^{\prime}\left(n_{2}\right)
$$

if

$$
n=\left(\begin{array}{cc}
n_{1} & u \\
0 & n_{2}
\end{array}\right)
$$

In particular, $\theta$ is a degenerate character. We define again the notion of a relevant orbit (with respect to the character $\theta$ ). If $\Phi$ is a smooth function of compact support on $S$ we will denote by $I(s, \Phi)$ its orbital integrals:

$$
I(s, \Phi)=\int \Phi\left({ }^{t} n s n\right) \theta(n) d n
$$

the integral is over $N$ divided by the fixator $N_{s}$ of $s$; by definition, the character $\theta$ is trivial in $N_{s}$, so that the integral makes sense. We think of this integral as a relative Kloosterman integral. Remarkably, the two kinds of Kloosterman integrals lead to the same kind of functions.

Let $w_{0}$ be the following matrix:

$$
w_{0}=\left(\begin{array}{cc}
0 & 1_{m} \\
1_{m} & 0
\end{array}\right)
$$

We will first show that every relevant orbit has a representative of the form

$$
s=w_{0}\left(\begin{array}{cc}
g^{\prime} & 0 \\
0 & -{ }^{t} g^{\prime}
\end{array}\right)
$$


where $g^{\prime}$ is in $G^{\prime}$ and the orbit of $g^{\prime}$ under $N^{\prime} \times N^{\prime}$ is relevant for $\theta^{\prime}$; furthermore, the orbit of $g^{\prime}$ determines the orbit of $s$ under $N$. Next, we will prove that given $\Phi$ there is $f^{\prime}$ such that the orbital integrals of $\Phi$ and $f$ match in the sense that:

$$
I(s, \Phi)=I\left(g^{\prime}, f^{\prime}\right)\left|\operatorname{det} g^{\prime}\right|^{(m-1) / 2},
$$

if $s$ and $g^{\prime}$ are as above. Although $f^{\prime}$ is not unique, there is a canonical choice for $f^{\prime}$ in terms of $\Phi$ : we will use the notation $\Phi \mapsto$ $f^{\prime}$ for this canonical choice.

Now let $\varepsilon$ be the following skew matrix:

$$
\varepsilon=\left(\begin{array}{cc}
0 & 1_{m} \\
-1_{m} & 0
\end{array}\right)
$$

and let $H$ be the fixator of $\varepsilon$ in $G$. For any smooth function of compact support $f$ on $G$, the function $\Phi_{f}$ on $S$ defined by:

$$
\Phi_{f}\left({ }^{t} g \varepsilon g\right)=\int_{H} f(h g) d h
$$

is smooth of compact support. Moreover, if $g$ is relevant, we have:

$$
\iint f(h g n) d h \theta(n) d n=I\left({ }^{t} g \varepsilon g, \Phi_{f}\right),
$$

the integral on the left is for $h \in H$ and $n$ in the quotient of $N$ by the fixator of ${ }^{t} g \varepsilon g$ in $N$, that is, the group

$$
N \cap g^{-1} H g \text {. }
$$

Consider now the function $f^{\prime}$ associated to $\Phi_{f}$. We will write

$$
f \mapsto f^{\prime} .
$$

If furthermore $f$ is a Hecke function then $f^{\prime}$ is also a Hecke function; we will show the map $f \mapsto f^{\prime}$ is an homomorphism of Hecke algebras. There is a dual correspondence on the set of unramified representations that we now describe: let $\pi^{\prime}$ be an unramified representation of $G^{\prime}$; consider then the parabolic subgroup of $P=M U$ of type $(m, m)$ in $G$ and the representation $\sigma$ of $G$ induced by the representation

$$
\left(\begin{array}{cc}
g_{1} & u \\
0 & g_{2}
\end{array}\right) \mapsto \pi^{\prime}\left(g_{1}\right)\left|\operatorname{det} g_{1}\right|^{1 / 2} \otimes \pi^{\prime}\left(g_{2}\right)\left|\operatorname{det} g_{2}\right|^{-1 / 2} .
$$

Let $\pi$ be its unique unramified constituent. We will show that the Satake transforms of these functions (see (19) below) are related by:

$$
\hat{f}(\pi)=\hat{f}^{\prime}\left(\pi^{\prime}\right) .
$$


Once these results are established, we can write down a global trace formula: let $F$ be a number field, and $f$ a smooth function of compact support on $G\left(F_{\mathrm{A}}\right)$; we will assume that $f$ is a product of functions $f_{v}$; for each place $v$, we will denote by $\Phi_{v}$ the corresponding function on $S_{v}$ and by $f_{v}^{\prime}$ the corresponding function on $G_{v}^{\prime}$ : thus $f_{v} \mapsto f_{v}^{\prime}$. We will denote by $f^{\prime}$ the product of the functions $f_{v}^{\prime}$. Now we can form the geometric kernels $K$ and $K^{\prime}$ attached to $f$ and $f^{\prime}$ respectively:

$$
K(x, y)=\sum_{\xi \in G(F)} f\left(x^{-1} \xi y\right), \quad K^{\prime}(x, y)=\sum_{\xi^{\prime} \in G^{\prime}(F)} f^{\prime}\left(x^{-1} \xi^{\prime} y\right) .
$$

Then we have the following identity

$$
\begin{aligned}
\iint K(h, n) d h \theta(n) d n \\
\quad=\iint K^{\prime}\left(n_{1}, n_{2}\right) \theta^{\prime}\left(n_{1}\right)^{-1} d n_{1} \theta^{\prime}\left(n_{2}\right) d n_{2}
\end{aligned}
$$

here

$$
h \in H(F) \backslash H\left(F_{\mathbb{A}}\right), \quad n \in N(F) \backslash N\left(F_{\mathbb{A}}\right), \quad n_{i} \in N^{\prime}(F) \backslash N^{\prime}\left(F_{\mathbb{A}}\right) .
$$

The right-hand side is the Kuznietsov trace formula and the left-hand side the relative Kuznietsov trace formula.

Indeed, the left-hand side can be written as:

$$
\sum_{\xi} \int f(h \xi n) \theta(n) d n
$$

where $h$ is in $H\left(F_{\mathbb{A}}\right), \xi$ in $H(F) \backslash G(F), n$ in $N(F) \backslash N\left(F_{\mathbb{A}}\right)$. Let $\Phi$ be the product of the functions $\Phi_{v}$. Then this expression can be expressed in terms of $\Phi$ as follows:

$$
\sum_{\sigma} \int \Phi\left({ }^{t} n \sigma n\right) \theta(n) d n \text {. }
$$

Here $\sigma$ is $S_{F}$ and $n$ is as before. We can also rewrite this integral with $\sigma$ in a set of representatives for the orbits of $N(F)$ on $S_{F}$ and $n$ in $N_{\sigma}(F) \backslash N\left(F_{\mathbb{A}}\right)$. For each $\sigma$ the integral factors through an integral

$$
\int_{N_{\sigma}(F) \backslash N_{\sigma}\left(F_{\mathrm{A}}\right)} \theta\left(n_{\sigma}\right) d n_{\sigma}
$$

and is thus zero unless $\theta$ is trivial on $N_{\sigma}$, that is, $\sigma$ is relevant. Thus we can rewrite our expression as

$$
\sum_{\sigma} \int \Phi\left({ }^{t} n \sigma n\right) \theta(n) d n
$$


where now $\sigma$ is in a set of representatives for the relevant orbits of $N(F)$ and $n$ in

$$
N_{\sigma}\left(F_{\mathrm{A}}\right) \backslash N\left(F_{\mathrm{A}}\right)
$$

This can be written also as a sum of global orbital integrals:

$$
\sum_{\sigma} I(\sigma, \Phi)
$$

each of which is a product of local ones. Next, we choose for $\sigma$ elements of the form:

$$
\sigma=w_{0}\left(\begin{array}{cc}
\tau & 0 \\
0 & -{ }^{t} \tau
\end{array}\right)
$$

where $\tau$ is in a set of representatives for the relevant orbits of $N^{\prime}(F) \times$ $N^{\prime}(F)$. Then our expression can be written also

$$
\sum_{\tau} I\left(\tau, f^{\prime}\right)
$$

since $|\operatorname{det} \tau|=1$. In turn, this is equal to the right-hand side of (11) and the identity (11) is proved.

It would be more difficult to prove a spectral version of this identity where $K$ and $K^{\prime}$ are replaced by the discrete spectrum kernels. Dual to this formula, would be a functorial map from the automorphic cuspidal representations of $G^{\prime}$ to the automorphic representations of $G$. The image would consist of automorphic representations of $G$ which are distinguished with respect to $H$, that is, which admit a vector $\phi$ such that the integral is non-zero:

$$
\int_{H(F) \backslash H\left(F_{\mathrm{A}}\right)} \phi(h) d h
$$

is non-zero. Moreover, if $\pi^{\prime}$ corresponds to $\pi$, then, for almost all $v$, the representation $\pi_{v}$ and $\pi_{v}^{\prime}$ are unramified and $\pi_{v}$ is the unramified component of the induced representation $\sigma_{v}$ (see (10)) determined by $\pi_{v}^{\prime}$. In fact, the image consists of residues of Eisenstein series and it is more convenient to study directly the above integral as in $[\mathbf{J}-\mathbf{R}]$.

Our motivation for studying this question is that we expect similar results for any symmetric space. For instance in $[\mathbf{J}-\mathbf{Y}]$, we begun to study the case where $S$ is the space of Hermitian matrices with respect to a quadratic extension $E$ of $F$ : in this case, $G=\mathrm{GL}(n, E)$, the character $\theta$ is generic and $G^{\prime}=\operatorname{GL}(n, F)$. The correspondence $\pi^{\prime} \mapsto \pi$ is the quadratic base change. The case at hand is much simpler, in fact elementary, and should serve as a model for the general case. 
2. Relevant orbits in $G^{\prime}$. We first recall the classification of relevant orbits for the action of $N^{\prime} \times N^{\prime}$ on $G^{\prime}$ (see [G]). Let $\Delta^{\prime}$ be the set of roots which are simple with respect to $A^{\prime} N^{\prime}$. Let $W^{\prime}$ be the Weyl group of $A^{\prime}$. We identify $W^{\prime}$ to the group of permutation matrices. For each root $\alpha$, we denote by $X_{\alpha}$ the corresponding root vector. Thus if $\alpha(a)=a_{i} / a_{j}, X_{\alpha}$ is the matrix with 1 in the $i$ th row and $j$ column entry and 0 in all other entries.

Suppose $w a$ is relevant. Let $\Theta^{\prime}$ be the set of simple roots $\alpha$ such that $w \alpha<0$ and let $\Theta^{\prime \prime}$ be the set of positive roots of the form $-w(\alpha)$ with $\alpha \in \Theta^{\prime}$. Then $\Theta^{\prime \prime}$ is contained in $\Delta^{\prime}$. Indeed, if $\beta=-w(\alpha)$ is not simple, we consider a pair $\left(n_{1}, n_{2}\right)$ with

$$
n_{1}=1+y X_{\beta}, \quad n_{2}=1+x X_{\alpha} .
$$

The relation

$$
{ }^{t} n_{1} w a n_{2}=w a
$$

is equivalent to

$$
y+\alpha(a) x=0 .
$$

Choose $x$ such that $\psi(x) \neq 0$ and then determine $y$ by the above equation. The pair $\left(n_{1}, n_{2}\right)$ is then in the fixator of $w a$ and yet $\theta^{\prime}\left(n_{1}\right) \theta^{\prime}\left(n_{2}\right)=\psi(x) \neq 1$, a contradiction.

Thus $\Theta^{\prime \prime}$ is contained in $\Delta^{\prime}$. Next, exchanging the roles of $w$ and $w^{-1}$ we see that $\Theta^{\prime \prime}$ is the set of simple roots $\alpha$ such that $w^{-1}(\alpha)<0$. We now appeal to a simple lemma that we will use again.

LEMMA 1. Let $w \in W^{\prime}$ and $\Theta^{\prime}$ (resp. $\Theta^{\prime \prime}$ ) the set of simple roots $\alpha$ such that $w \alpha<0$ (resp. $\left.w^{-1} \alpha<0\right)$. Suppose $w\left(\Theta^{\prime}\right)=-\Theta^{\prime \prime}$. Then, in fact $\Theta^{\prime}=\Theta^{\prime \prime}$ and $w^{2}=1$. Furthermore, let $P^{\prime}=M^{\prime} U^{\prime}$ be the standard parabolic subgroup determined by $\Theta^{\prime}$. Then $w$ is in $M^{\prime}$ and is the longest element of $W \cap M^{\prime}$.

Here standard means that $P^{\prime}$ contains $A^{\prime} N^{\prime}$; the Levi-factor $M^{\prime}$ is the one containing $A^{\prime}$; thus $M^{\prime}$ is determined by the condition that the roots $\alpha$ in $\Theta^{\prime}$ be "contained" in $M^{\prime}$ (i.e. the root group $N_{\alpha}$ is contained in $M^{\prime}$ ). For the proof, we introduce also the parabolic subgroup $P^{\prime \prime}=M^{\prime \prime} U^{\prime \prime}$ determined by $\Theta^{\prime \prime}$. Clearly, $w M^{\prime} w^{-1}=M^{\prime \prime}$. Next we claim any root $\gamma$ in $U^{\prime}$ is transformed into a positive root in $U^{\prime \prime}$ by $w$. Indeed, $\beta=w \gamma$ cannot be in $M^{\prime \prime}$. Otherwise, applying $w^{-1}$ to $\beta$ we would find that $\gamma$ is in $M^{\prime}$. Now suppose $\beta$ is in $\bar{U}^{\prime \prime}$. Then

$$
\beta=-\sum_{\alpha \in \Delta} n_{\alpha} \alpha
$$


with $n_{\alpha} \geq 0$ for all $\alpha$ and $n_{\alpha}>0$ for at least one $\alpha \notin \Theta^{\prime \prime}$. On the other hand,

$$
\gamma=\sum_{\alpha \in \Delta} m_{\alpha} \alpha
$$

with $m_{\alpha} \geq 0$ for all $\alpha$ and $m_{\alpha}>0$ for at least one $\alpha \notin \Theta^{\prime}$. Applying $w$ to this relation we get

$$
\beta=w \gamma=-\sum_{\alpha \in \Theta^{\prime}} m_{\alpha}(-w \alpha)+\sum_{\alpha \notin \Theta^{\prime}} m_{\alpha} w \alpha .
$$

Since $\alpha \mapsto-w \alpha$ is a bijection of $\Theta^{\prime}$ onto $\Theta^{\prime \prime}$, we get that

$$
\sum_{\alpha \notin \Theta^{\prime}} m_{\alpha} w \alpha=\sum_{\alpha \in \Theta^{\prime \prime}} n_{\alpha}^{\prime} \alpha-\sum_{\alpha \notin \Theta^{\prime \prime}} n_{\alpha} \alpha,
$$

for suitable $n_{\alpha}^{\prime}$. The left-hand side is a non-zero sum of positive roots, hence a linear combination of simple roots with positive coefficients, one of which is strictly positive. However, this is not the case for the right-hand side and we get a contradiction. We conclude that $w \gamma$ is in $U^{\prime \prime}$ for $\gamma$ in $U^{\prime}$. Hence $w$ transforms $P^{\prime}$ into $P^{\prime \prime}$. Since both are standard we must have $P^{\prime}=P^{\prime \prime}$ and $\Theta^{\prime}=\Theta^{\prime \prime}$. Thus $w$ is in $M^{\prime}$ and $w \Theta^{\prime}=-\Theta^{\prime}$. In other words, $w$ is the longest element of $W \cap M^{\prime}$. This concludes the proof of the lemma.

The lemma being proved, we apply it to our relevant element wa. Thus $w$ is the longest element in $W \cap M^{\prime}$, where $P^{\prime}=M^{\prime} U^{\prime}$ is determined by $\Theta^{\prime}$. It remains to see that $a$ is in the center of $M^{\prime}$. Let $\alpha$ be a root in $\Theta^{\prime}$ and set $\beta=-w \alpha$. Consider elements $\left(n_{1}, n_{2}\right)$ with

$$
n_{1}=1+y X_{\beta}, \quad n_{2}=1+x X_{\alpha} .
$$

The relation

$$
{ }^{t} n_{1} w a n_{2}=w a
$$

is again equivalent to

$$
y+\alpha(a) x=0
$$

but now

$$
\theta^{\prime}\left(n_{1} n_{2}\right)=\psi(x+y)
$$

We see that

$$
y+\alpha(a) x=0,
$$

must imply $\psi(x+y)=1$. This means that $\alpha(a)=1$. Thus $a$ is indeed in the center of $M^{\prime}$.

In conclusion, we have proved the following result: 
Proposition 1. Let $M^{\prime}$ be a standard parabolic subgroup of $G^{\prime}, w$ the longest element in $W \cap M^{\prime}$ and $a$ in the center of $M^{\prime}$. Then wa is relevant. All relevant elements have this form.

3. Relevant orbits in $S$. We first describe all orbits of $N$ on $S$ :

LEMMA 2 ([S]). Every $s \in S$ can be written in the form

$$
s={ }^{t} \text { nwan }
$$

with $n \in N, a \in A, w \in W, w^{2}=1$ and $w a w=-a$.

Indeed, every element can be written in the form

$$
s={ }^{t} n_{1} w_{a n_{2}}
$$

with $n_{i} \in N$. Since ${ }^{t} s=-s$ and $w$ and $a$ are uniquely determined, we have ${ }^{t}(w a)=-w a$. Since ${ }^{t} w=w^{-1}$ we get the condition of the lemma for $w a$. Next, we let $N^{+}$(resp. $N^{-}$) be the group generated by the root groups $N_{\alpha}$ with $\alpha>0$ and $w \alpha>0$ (resp. $w \alpha<0$ ). We have $N=N^{+} N^{-}=N^{-} N^{+}$. Let us write

$$
n_{1}=n_{1}^{-} n_{1}^{+}, \quad n_{2}=n_{2}^{+} n_{2}^{-} \text {. }
$$

Replacing $s$ by ${ }^{t}\left(n_{2}^{-}\right)^{-1} s\left(n_{2}^{-}\right)^{-1}$, we may assume $n_{2} \in N^{+}$. We have then

$$
s=-{ }^{t} s={ }^{t} n_{2} w a n_{1}^{-} n_{1}^{+}
$$

and so $n_{1}^{+}=n_{2}$. Replacing $s$ by an element of the same orbit, we see we may assume $n_{2}=1, n_{1}^{+}=1$. In other words:

$$
s={ }^{t} n_{1} w a
$$

with $n_{1} \in N^{-}$. Writing once more that $s$ is skew symmetric we find:

$$
{ }^{t} n_{1}=\operatorname{wan}_{1} a^{-1} w^{-1} \text {. }
$$

For $n \in N^{-}$, let us write

$$
\xi(n)=w a^{-1}\left({ }^{t} n^{-1}\right) a w^{-1} .
$$

Since $w^{2}=1, \xi(n)$ is again in $N^{-}$and $\xi^{2}=1$. Thus $\xi$ is an automorphism of $N^{-}$of order 2. Now the above condition reads $\xi\left(n_{1}\right)=n_{1}^{-1}$. Since $N$ is nilpotent there is $n \in N^{-}$such that

$$
n_{1}=\xi(n)^{-1} n \text {. }
$$

This condition reads

$$
s={ }^{t} \text { nwan }
$$

and we are done.

We first consider the case of a generic character. Thus we temporarily define $\theta$ by formula (1) with $m$ replaced by $2 m$. Then: 
PROPOSITION 2. Suppose $\theta$ is a generic character of $N$. Then there are no relevant orbits for $\theta$ in $S$.

We first observe the following: if $\alpha$ is a simple root and $w \alpha$ is negative, then $w \alpha=-\beta$ with $\beta \in \Delta$. Indeed, suppose $\beta$ is not a simple root. We choose root vectors $X_{\gamma}$ in the usual way but agree that $X_{\alpha+\beta}=0$ if $\alpha+\beta$ is not a root. Define

$$
n=1+x X_{\alpha}+y X_{\beta}+z X_{\alpha+\beta} .
$$

Then

$$
\begin{aligned}
{ }^{t} \text { nwana }^{-1} w^{-1}= & +(x+y \beta(a)) X_{-\alpha}+(y+x \alpha(a)) X_{-\beta} \\
& +(z(1+\alpha(a) \beta(a))+u) X_{-\alpha-\beta},
\end{aligned}
$$

where $u$ depends only on $(x, y)$ but not on $z$. We have

$$
\theta(n)=\psi(x) \text {. }
$$

On the other hand,

$$
\beta(a)=(w \alpha(a))^{-1}=\alpha\left(w a w^{-1}\right)^{-1}=\alpha(-a)^{-1}=\alpha(a)^{-1} .
$$

Thus, if

$$
\psi(x) \neq 1, \quad y=-\alpha(a) x, \quad z=-\frac{u}{2}
$$

then $n$ is in the fixator of $w a$ and yet $\theta(n) \neq 1$, a contradiction. Thus $\beta$ is in $\Delta$. Let $\Theta$ be the set of simple roots $\alpha$ such that $w(\alpha)<0$. Then $w(\boldsymbol{\Theta})=-\Theta^{\prime}$, with $\Theta^{\prime}$ contained in $\Delta$. Since $w^{2}=1$, we have $\Theta^{\prime} \subseteq \Theta$ and thus $\Theta^{\prime}=\Theta$. Let $P=M U$ be the parabolic subgroup determined by $\Theta$. Then, by Lemma $1, w$ is the longest element in $W \cap M$.

Finally, we consider again a root $\alpha$ in $\Theta$ and the root $\beta=-w \alpha$. Consider $n$ as in (12), then

$$
\theta(n)=\psi(x+y) .
$$

Thus $\psi(x+y)=1$ if $y=-x \alpha(a)$; this implies $\alpha(a)=1$.

We now derive a contradiction. It will be convenient to identify $W$ to the group or permutations of the set $[1,2 m]$; for each $i$ we will denote by $\alpha_{i}$ the simple root $a \mapsto a_{i} / a_{i+1}$. Since $w$ is the longest element of $W \cap M$, there is an index $i$ such that $w i=i$ or $w i=i+1$. In the first case, the relation $w a=-a$ implies $a_{i}=-a_{i}$ or $a_{i}=0$, a contradiction. In the second case, we get $a_{i}=-a_{i+1}$, that is, $\alpha_{i}(a)=-1$. However, we also have $w \alpha_{i}=-\alpha_{i}$ so $\alpha_{i}$ is in $\Theta$; then $\alpha_{i}(a)=1$, a contradiction. Thus there is no relevant orbit in this case. An essentially equivalent result is the following: 
Proposition 3. If $\theta$ is a generic character of $N$ then there is no non-zero distribution on $G$ which is invariant on the left under $H$ and transforms on the right under $\theta$.

We sketch a proof. Any distribution on $G$ invariant under $H$ may be viewed as a distribution on $S$. Thus we have to prove there is no distribution $\mu$ on $S$ such that

$$
\int f\left({ }^{t} n s n\right) d \mu(s)=\theta(n) \int f(s) d \mu(s) .
$$

For each $w$ in $W$ with $w^{2}=1$, let $S_{w}$ be the set of matrices of the form ${ }^{t}$ nwan with waw $=-a$. We can write $S$ as a finite union of an increasing sequence of open subsets $X_{i}$ such that each difference $X_{i+1}-X_{i}$ is one of the sets $S_{w}$. We prove by induction on $i$ that there is no non-zero distribution on $X_{i}$ which transforms under $\theta$. Assuming our assertion proved for $i$, we prove it for $i+1$. Consider the space $V$ of distributions on $X_{i+1}$ which transform under $\theta$. The restriction of such a distribution to $X_{i}$ is zero by assumption. Thus, we may view $V$ as the space of distributions on the manifold $S_{w}$ which transform under $\theta$. Let $T$ be the set of $a$ in $A$ such that $w a w=-a$. Then $T$ is a manifold and there is a projection map

$$
p: S_{w} \rightarrow T
$$

defined by:

$$
p\left({ }^{t} n w a n\right)=a .
$$

We use it to regard the space of distributions on $S_{w}$ as a module over the space of smooth functions on $T$. In particular, $V$ is a submodule. By the localisation principle of Gel'fand-Kazhdan (see [B]), the elements of $V$ which are supported by a fiber form a total subspace in $V$, that is, their linear combinations are dense in $V$ for the weak topology. However, a fiber may be identified to the quotient of $N$ by the fixator $N_{s}$ of a point $s \in S$. A distribution supported by the fiber may be viewed as a distribution on this quotient. Since $\theta$ is non-trivial on $N_{s}$, a distribution on $N_{s} \backslash N$ which transforms under $\theta$ is necessarily 0 and we are done.

We note that, in turn, this implies that a generic representation of $G$ does not have a symplectic model, that is, does not imbed into the space of functions on $H \backslash G$ ([H-R]).

We now turn to the case of the degenerate character $\theta$ determined by formula (5). 
Proposition 4. Among the elements of the system of representatives of Lemma 2, the ones relevant for $\theta$ are those of the form

$$
s=w_{0}\left(\begin{array}{cc}
w^{\prime} a^{\prime} & 0 \\
0 & -w^{\prime} a^{\prime}
\end{array}\right)
$$

where $w^{\prime}$ is in $W^{\prime}, a^{\prime}$ in $A^{\prime}$ and $w^{\prime} a^{\prime}$ is relevant in $G^{\prime}$ for $\theta^{\prime}$.

First, we check that such an element is indeed relevant. Suppose that

$$
n=\left(\begin{array}{cc}
n_{1} & u \\
0 & n_{2}
\end{array}\right)
$$

fixes $s$. Then $\left(n_{1}, n_{2}\right)$ fixes $w^{\prime} a^{\prime}$. Hence

$$
\theta(n)=\theta^{\prime}\left(n_{1}\right) \theta^{\prime}\left(n_{2}\right)=1 \text {. }
$$

So $s$ is relevant.

Conversely, let us show that every relevant element $w a$ has this form. We first observe the following: if $\alpha$ is a simple root not equal to $\alpha_{m}$ and $w \alpha$ is negative, then $w \alpha=-\beta$ with $\beta \in \Delta-\left\{\alpha_{m}\right\}$. Indeed, since $\theta$ is trivial on $N_{\alpha_{m}}$ and non-trivial on any $N_{\alpha}$ with $\alpha \neq \alpha_{m}$, the proof is the same as before. Moreover, just as before, $\alpha(a)=1$ for such a root. Let $\Theta_{1}$ be the set of simple roots $\alpha \neq \alpha_{m}$ such that $w \alpha<0$. Then $w \Theta_{1}=-\Theta^{\prime}$ where $\Theta^{\prime}$ is a suitable subset of $\Delta-\left\{\alpha_{m}\right\}$. Since $w^{2}=1$, we have $\Theta^{\prime} \subseteq \Theta_{1}$ and thus $\Theta^{\prime}=\Theta_{1}$ or

$$
w \Theta_{1}=-\Theta_{1} \text {. }
$$

Now suppose that $w \alpha_{m}>0$. Then $\Theta_{1}$ is the set of simple roots $\alpha$ such that $w \alpha<0$. Let $P_{1}=M_{1} U_{1}$ be the parabolic subgroup attached to $\Theta_{1}$. Then by Lemma $1 w$ is the longest element of $W \cap M_{1}$. As before, this gives a contradiction: either there is an $i$ such that $w i=i$ or there is a root $\alpha$ in $\Theta_{1}$ such that $w \alpha=-\alpha$. In the first case, we get $a_{i}=0$; in the second case, we get $\alpha(a)=-1$. In either case, we get a contradiction.

Thus $w \alpha_{m}<0$. Let $\Theta$ be the set $\Delta-\left\{\alpha_{m}\right\}$ and $\Theta_{2}$ be the set $\Delta-\Theta_{1}-\left\{\alpha_{m}\right\}$. Let $P=M U$ be the parabolic subgroup attached to $\Theta$. Suppose $w \alpha_{m}$ is in $M$. Then:

$$
w \alpha_{m}=-\sum_{\Theta_{1}} n_{\alpha} \alpha-\sum_{\Theta_{2}} n_{\alpha} \alpha,
$$

with $n_{\alpha} \geq 0$. Applying $w$ to this formula, we get:

$$
\alpha_{m}+\sum_{\Theta_{2}} n_{\alpha} w \alpha=\sum_{\Theta_{1}} n_{\alpha}(-w \alpha) \text {. }
$$


Since $\alpha \mapsto-w \alpha$ is a bijection of $\Theta_{1}$ onto itself, and $w \alpha>0$ for $\alpha \in \Theta_{2}$, this implies $\alpha_{m} \in \Theta_{1}$, a contradiction. We conclude $w \alpha_{m}$ is in $\bar{U}$.

Next, let $\alpha_{i}$ be a root in $\Theta_{2}$. Thus $w \alpha_{i}>0$. We are going to show that $w \alpha_{i}$ is in $M$. Suppose it is in $U$. Then if $i+1 \leq m$, we have $w i \leq m$ and $w(i+1)>m$. If $m<i$, then $w i \leq m, w(i+1)>$ $m$. Let us show that the first case will lead to a contradiction. The argument would be similar for the second case. We have found the existence of an integer $j$ such that $j<m$ and $w j \leq m$; let us assume $j$ is the least such integer. Then $j=1$. Otherwise, we would have $w(j-1)>m$ and the root $\alpha_{j-1}$ would be changed by $w$ into a negative root in $\bar{U}$. However, by definition of $\Theta_{1}, \alpha_{j-1}$ would be in $\Theta_{1}$ and $w\left(\alpha_{j-1}\right)$ would be in $M_{1} \subseteq M$, a contradiction. We thus have $k=w 1 \leq m$. We now claim that $w$ transforms the interval $[1, k]$ into itself and reverses its order, that is, for $1 \leq i \leq k$, we have $i+w i=k+1$. We have just proved this for $i=1$. Assume it is true for $1,2, \ldots, l$ with $2 l \leq k$; if $2 l=k$ or $2 l+1=k$ we are done. So we assume this is not the case and we prove our assertion for $l+1$. We have in particular, $w l=k-l+1$. Consider $w(k-l)$. Then $w(k-l)>l=w(k-l+1)$. Thus $w \alpha_{k-l}$ is a negative root; it follows that $\alpha_{k-l}$ is in $\Theta_{1}$ and $w \alpha_{k-l}$ is the opposite of a simple root. This implies that $w(k-l)=l+1$. Thus we obtain our assertion by induction on $l$. Now if $k$ is odd, we get $w((k+1) / 2)=(k+1) / 2$ and $a_{(k+1) / 2}=0$, a contradiction. If $k$ is even then $w \alpha_{k / 2}=-\alpha_{k / 2}$ and $\alpha_{k / 2}$ is in $\Theta_{1}$ with $\alpha_{k / 2}(a)=-1$. Again, this is a contradiction. We conclude that $w \alpha_{i}$ is in $M$.

At this point, we have

$$
w \Theta_{1}=-\Theta_{1}, \quad w \Theta_{2}>0, \quad w\left(\Theta_{2}\right) \subseteq M, \quad w\left(\alpha_{m}\right) \in \bar{U} .
$$

Now consider the element $w_{1}=w_{0} w$, where $w_{0}$ is defined in (7). Since $w_{0}$ takes $\Theta$ to itself and $U$ to $\bar{U}$, we have

$$
\begin{aligned}
& w_{1} \Theta_{1}=-\Theta^{\prime}, \quad \text { with } \Theta^{\prime} \subseteq \Theta, \\
& w_{1} \Theta_{2}>0, \quad w_{1}\left(\Theta_{2}\right) \subseteq M .
\end{aligned}
$$

On the other hand,

$$
w_{1} \alpha_{m} \in U \text {. }
$$

Let $P^{\prime}=M^{\prime} U^{\prime}$ be the parabolic subgroup determined by $\Theta^{\prime}$. Recall $P_{1}$ is the one determined by $\Theta_{1}$. We have $w_{1} M_{1} w_{1}^{-1}=M^{\prime}$. Next, if $\beta$ is in $U_{1}$, then

$$
\beta=\sum_{\Delta} n_{\alpha} \alpha
$$


with $n_{\alpha} \geq 0$ for all $\alpha$ and $n_{\alpha}>0$ for $\alpha=\alpha_{m}$ or some $\alpha \in \Theta_{2}$. This implies that $\gamma=w_{1} \beta$ is positive. Indeed:

$$
\gamma+\sum_{\Theta_{1}} n_{\alpha} w_{1}(-\alpha)=\sum_{\Theta_{2}} n_{\alpha} w_{1} \alpha+n_{\alpha_{m}} w_{1} \alpha_{m} .
$$

The right-hand side is a sum of positive roots. Moreover $\alpha \mapsto w_{1}(-\alpha)$ is a bijection of $\Theta_{1}$ onto $\Theta^{\prime}$. Thus if $\gamma$ is negative it must be in $M^{\prime}$. Applying $w_{1}^{-1}$ to $\gamma$, we would find $\beta$ is in $M_{1}$, a contradiction. Thus $\gamma$ is positive and in fact in $U^{\prime}$. We conclude that $w_{1} P_{1} w_{1}^{-1}$ is contained in $P^{\prime}$, hence equal to it. Since $P_{1}$ and $P^{\prime}$ are standard parabolic subgroups, it follows that $P_{1}=P^{\prime}, \Theta_{1}=\Theta^{\prime}$ and $w_{1}=w_{0} w$ transforms $\Theta_{1}$ into $-\Theta_{1}$. Thus $w_{1}$ is in fact the longest element of $W \cap M_{1}$. We have also $w_{0} \Theta_{1}=\Theta_{1}$. This means that there is a Levisubgroup $M^{\prime}$ of $G^{\prime}=\mathrm{GL}(m)$ such that $M_{1}$ is the group of matrices of the form

$$
\left(\begin{array}{cc}
m_{1} & 0 \\
0 & m_{2}
\end{array}\right)
$$

with $m_{i} \in M^{\prime}$ and

$$
w_{1}=\left(\begin{array}{cc}
w^{\prime} & 0 \\
0 & w^{\prime}
\end{array}\right)
$$

where $w^{\prime}$ is the longest element of $W^{\prime} \cap M^{\prime}$. Finally, the conditions $\alpha(a)=1$ for $\alpha$ in $\Theta_{1}$ and $w(a)=-a$ mean that $a$ has the form:

$$
\left(\begin{array}{cc}
a^{\prime} & 0 \\
0 & -a^{\prime}
\end{array}\right)
$$

where $a^{\prime}$ is in the center of $M^{\prime}$. So

$$
w a=w_{0}\left(\begin{array}{cc}
w^{\prime} a^{\prime} & 0 \\
0 & -w^{\prime} a^{\prime}
\end{array}\right),
$$

and the proposition is completely proved.

4. Matching orbital integrals. Let $\Phi$ be a smooth function of compact support on $S$. We will set

$$
f^{\prime}(g)=\int \Phi\left[w_{0}\left(\begin{array}{cc}
g & v \\
0 & -{ }^{t} g
\end{array}\right)\right] d v|\operatorname{det} g|^{(1-m) / 2} .
$$

Here $v$ is integrated over the vector space $\operatorname{Sk}(m \times m)$ of skew $m \times m$ matrices. The Haar measure is self dual when we identify that vector space to its dual, via

$$
\left(v, v^{\prime}\right) \mapsto \frac{1}{2} \operatorname{Tr}\left(v v^{\prime}\right) .
$$

We will write $\Phi \mapsto f^{\prime}$. Clearly, $f^{\prime}$ is a smooth function of compact support on $G^{\prime}$. We claim that $\Phi$ and $f^{\prime}$ have matching orbital integrals: 
Proposition 5. Let $g$ be a relevant element in $G^{\prime}$ and set

$$
s=w_{0}\left(\begin{array}{cc}
g & 0 \\
0 & -{ }^{t} g
\end{array}\right) .
$$

Then

$$
I(s, \Phi)=J\left(g, f^{\prime}\right)|\operatorname{det} g|^{(m-1) / 2} .
$$

The proof of the proposition will indicate the correct choice of the Haar measures. Consider the orbital integral

$$
I(s, \Phi)=\int \Phi\left({ }^{t} n s n\right) \theta(n) d n
$$

Let us write:

$$
n=\left(\begin{array}{ll}
1 & u \\
0 & 1
\end{array}\right)\left(\begin{array}{cc}
n_{1} & 0 \\
0 & n_{2}
\end{array}\right)
$$

Then

$$
{ }^{t} n s n=w_{0}\left(\begin{array}{cc}
{ }^{t} n_{2} g n_{1} & { }^{t} n_{2} A_{g}(u) n_{2} \\
0 & -{ }^{t} n_{1}{ }^{t} g n_{2}
\end{array}\right)
$$

where we have set:

$$
A_{g}(u)=g u-{ }^{t} u^{t} g .
$$

Note that the image of $A_{g}$ is the space $\operatorname{Sk}(m \times m)$. We see that $n$ is in the fixator $N_{s}$ of $s$ if and only if $u$ is in the kernel of $A_{g}$ and the pair $\left(n_{1}, n_{2}\right)$ in the fixator $N_{g}^{\prime}$ of $g$ in $N^{\prime} \times N^{\prime}$. Moreover, $N_{s} \backslash N$ is isomorphic to the product

$$
\left(N_{g}^{\prime} \backslash N^{\prime} \times N^{\prime}\right) \times\left(\operatorname{ker} A_{g} \backslash M(m \times m)\right) .
$$

Let us choose a measure on the quotient $N_{g}^{\prime} \backslash N^{\prime} \times N^{\prime}$ and use $A_{g}$ to transport the measure on $\operatorname{Sk}(m \times m)$ to

$$
\operatorname{ker} A_{g} \backslash M(m \times m) \text {. }
$$

We obtain then a measure on $N_{s} \backslash N$. For that measure, we get:

(18) $I(s, \Phi)$

$$
=\iint \Phi\left[w_{0}\left(\begin{array}{cc}
{ }^{t} n_{2} g n_{1} & { }^{t} n_{2} v n_{2} \\
0 & -{ }^{t} n_{1}{ }^{t} g n_{2}
\end{array}\right)\right] d v \theta^{\prime}\left(n_{1} n_{2}\right) d n_{1} d n_{2} .
$$

Here $v$ is integrated over $\operatorname{Sk}(m \times m)$. After changing $v$ to ${ }^{t} n_{2} v n_{2}$, we obtain

$$
I(s, \Phi)=\iint \Phi\left[w_{0}\left(\begin{array}{cc}
{ }^{t} n_{2} g n_{1} & v \\
0 & -{ }^{t} n_{1}{ }^{t} g n_{2}
\end{array}\right)\right] d v \theta^{\prime}\left(n_{1} n_{2}\right) d n_{1} d n_{2}
$$


or

$$
\begin{aligned}
I(s, \Phi) & =\int f^{\prime}\left({ }^{t} n_{1} g n_{2}\right) \theta^{\prime}\left(n_{1} n_{2}\right) d n_{1} d n_{2}|\operatorname{det} g|^{(m-1) / 2} \\
& =J\left(g, f^{\prime}\right)|\operatorname{det} g|^{(m-1) / 2} .
\end{aligned}
$$

Thus, we have proved the proposition.

Now we discuss orbital integrals for Hecke functions. We assume $F$ is non-Archimedean of odd residual characteristic. Let $R$ be the ring of integers of $F$. We choose a character $\psi$ with conductor $R$ and set $K=\operatorname{GL}(2 m, R), K^{\prime}=\mathrm{GL}(m, R)$. Suppose that $f$ is a Hecke function, that is, a function of compact support, bi-invariant under $K=\mathrm{GL}\left(2 m, R_{F}\right)$. As in the introduction, define:

$$
\Phi_{f}\left({ }^{t} g \varepsilon g\right)=\int f(h \varepsilon g) d h,
$$

where $H$ is the fixator of $\varepsilon$ in $K$. It is easy to see that $K \cap S$ is one orbit of $K$. Because $f$ is $K$-invariant on the left, we could replace $\varepsilon$ by any other element of $K \cap S$ in this definition and yet arrive at the same function $\Phi_{f}$. On the other hand, the invariance of $f$ under $K$ on the right implies that $\Phi_{f}$ is invariant under $K$. For the function $f^{\prime}$ corresponding to $\Phi_{f}$, this implies that $f^{\prime}$ is bi- $K^{\prime}$-invariant.

We claim that the map $f \mapsto f^{\prime}$ is an homomorphism of the Hecke algebras. First suppose that $f$ is the characteristic function of $K$. Then $\Phi_{f}(s)=0$ unless $s$ is in the orbit of $\varepsilon$ under $K$, that is, is in $K \cap S$. For such an $s$, we have $\Phi_{f}(s)=1$. Thus $\Phi_{f}$ is the characteristic function of $K \cap S$. This implies that the function $f^{\prime}$ is the characteristic function of $K^{\prime}$.

Now let $\pi^{\prime}$ be an unramified irreducible representation of $G_{m}$. Let $V^{\prime}$ be its space, $\omega^{\prime}$ the corresponding spherical function. Denote by $f^{\prime} \mapsto \hat{f}^{\prime}\left(\pi^{\prime}\right)$ the corresponding character of the Hecke algebra:

$$
\int_{G^{\prime}} f^{\prime}\left(g^{\prime}\right) \omega^{\prime}\left(g^{\prime}\right) d g^{\prime}=\hat{f}^{\prime}\left(\pi^{\prime}\right) \text {. }
$$

Recall that the representation $\pi^{\prime}$ is contragredient to the representation

$$
g \mapsto \pi^{\prime}\left({ }^{t} g^{-1}\right)
$$

In other words, there is a linear form $\beta \neq 0$ on the space $V^{\prime} \otimes V^{\prime}$ such that

$$
\beta\left(\pi^{\prime}(g) \otimes \pi^{\prime}\left({ }^{t} g^{-1}\right) v\right)=\beta(v) .
$$

We choose a $K^{\prime}$-invariant vector $\phi_{0}^{\prime}$ in the representation such that

$$
\beta\left(\phi_{0}^{\prime} \otimes \phi_{0}^{\prime}\right)=1 \text {. }
$$


We use $\pi^{\prime}$ to construct a representation $\sigma$ of $G$ induced from the parabolic subgroup $P$ of type $(m, m)$. Its space is the space $V$ of smooth functions $\phi$ on $G$ with values in the space $V^{\prime} \otimes V^{\prime}$ such that

$$
\phi\left[\left(\begin{array}{cc}
g_{1} & x \\
0 & g_{2}
\end{array}\right) g\right]=\left|\frac{\operatorname{deg} g_{1}}{\operatorname{det} g_{2}}\right|^{(m+1) / 2} \pi^{\prime}\left(g_{1}\right) \otimes \pi^{\prime}\left(g_{2}\right) \phi(g) \text {. }
$$

Let $\phi_{0}$ be the function in that space such that

$$
\phi_{0}(k)=\phi_{0}^{\prime}, \quad k \in K \text {. }
$$

In particular, $\phi_{0}$ is $K$-invariant. We let $\pi$ be the irreducible component of $\sigma$ containing the unit representation of $K$. Our aim is to prove the following result:

Proposition 6. With the above notations,

$$
\hat{f}(\pi)=\hat{f}^{\prime}\left(\pi^{\prime}\right) \text {. }
$$

In particular, the map $f \mapsto f^{\prime}$ is a morphism of the Hecke algebras.

Before proving the proposition, we remark it is the fundamental lemma for the case at hand: we could define $f^{\prime}$ by this property. Then the proposition states that the orbital integrals of $f$ and $f^{\prime}$ match:

$$
\begin{aligned}
& \int f(h g n) d h \theta(n) d n \\
& \quad=\left|\operatorname{det} g^{\prime}\right|^{(m-1) / 2} \iint f^{\prime}\left({ }^{t} n_{1} g^{\prime} n_{2}\right) \theta^{\prime}\left(n_{1}\right) \theta^{\prime}\left(n_{2}\right) d n_{1} d n_{2}
\end{aligned}
$$

where $g^{\prime}$ is relevant for $\theta^{\prime}$ in $G^{\prime}$ and $g$ is such that

$$
{ }^{t} g \varepsilon g=w_{0}\left(\begin{array}{cc}
g^{\prime} & 0 \\
0 & -{ }^{t} g^{\prime}
\end{array}\right) \text {. }
$$

As in [H-R], we construct a linear form $\gamma$ on $V$ which is invariant under translation by $H$. The group $P \cap H$ is a parabolic subgroup in $H$. A Levi factor consists of all matrices of the form

$$
m=\left(\begin{array}{cc}
g & 0 \\
0 & t \\
g^{-1}
\end{array}\right) \text {. }
$$

The unipotent radical consists of all matrices of the form

$$
\left(\begin{array}{ll}
1 & u \\
0 & 1
\end{array}\right)
$$

where ${ }^{t} u=u$. The module of $P \cap H$ is given by:

$$
\delta_{P \cap H}(m)=|\operatorname{det} g|^{m+1} \text {. }
$$


Thus the function $g \mapsto \beta(\phi(g))$ satisfies

$$
\beta(\phi(p g))=\delta_{P \cap H}(p) \beta(\phi(g)),
$$

for $p \in P \cap H$. In particular, the linear form

$$
\gamma(\phi)=\int_{K \cap H} \beta(\phi(k)) d k
$$

has the required invariance property:

$$
\gamma(\sigma(h) \phi)=\gamma(\phi), \quad h \in H .
$$

Moreover

$$
\gamma\left(\phi_{0}\right)=1
$$

Now suppose $f$ is in the Hecke algebra $G$. Then we have:

$$
\int \phi_{0}(g x) f(x) d x=\phi_{0}(g) \hat{f}(\pi) \text {. }
$$

Let us apply the linear form $\gamma$ to both sides. We obtain

$$
\hat{f}(\pi)=\beta\left[\iint \phi_{0}(k x) f(x) d x d k\right]
$$

the integral over $G \times(K \cap H)$. After a change of variables, the integral on the right can be written

$$
\hat{f}(\pi)=\beta\left[\iint \phi_{0}(x) f(k x) d x d k\right] .
$$

Set

$$
x=\left(\begin{array}{cc}
g & 0 \\
0 & { }^{t} g^{-1}
\end{array}\right)\left(\begin{array}{cc}
1 & u \\
0 & 1
\end{array}\right)\left(\begin{array}{cc}
1 & v \\
0 & 1
\end{array}\right)\left(\begin{array}{cc}
g^{\prime} & 0 \\
0 & 1
\end{array}\right) k_{1}
$$

where $u$ is symmetric, $v$ skew symmetric and $k_{1} \in K$. Then

$$
d x=d g d g^{\prime} d u d v d k_{1}\left|\operatorname{det} g^{\prime}\right|^{-m} .
$$

Our integral becomes

$$
\begin{aligned}
& \int f\left[k\left(\begin{array}{cc}
g & 0 \\
0 & { }^{t} g^{-1}
\end{array}\right)\left(\begin{array}{ll}
1 & u \\
0 & 1
\end{array}\right)\left(\begin{array}{ll}
1 & v \\
0 & 1
\end{array}\right)\left(\begin{array}{ll}
g^{\prime} & 0 \\
0 & 1
\end{array}\right)\right] \\
& \cdot \beta\left[\pi^{\prime} \otimes \pi^{\prime}\left(\begin{array}{ll}
g^{\prime} & 0 \\
0 & 1
\end{array}\right) \phi_{0}\right]\left|\operatorname{det} g^{\prime}\right|^{(1-m) / 2}|\operatorname{det} g|^{m+1} d g d g^{\prime} d u d v d k \text {. }
\end{aligned}
$$


We recognize the Haar measure $d h$ on $H$ and we get:

$$
\int f\left[h\left(\begin{array}{ll}
1 & v \\
0 & 1
\end{array}\right)\left(\begin{array}{ll}
g^{\prime} & 0 \\
0 & 1
\end{array}\right)\right] \beta\left(\pi^{\prime}\left(g^{\prime}\right) \phi_{0}^{\prime} \otimes \phi_{0}^{\prime}\right)\left|\operatorname{det} g^{\prime}\right|^{(1-m) / 2} d h d g^{\prime} d v \text {. }
$$

Now

$$
\beta\left(\pi^{\prime}\left(g^{\prime}\right) \phi_{0}^{\prime} \otimes \phi_{0}^{\prime}\right)
$$

is the spherical function $\omega^{\prime}$ attached to $\pi^{\prime}$. In terms of $\Phi_{f}$ the previous integral can be written as

$$
\begin{gathered}
\int \Phi_{f}\left[\left(\begin{array}{cc}
g^{\prime} & 2 v \\
0 & -{ }^{t} g^{\prime}
\end{array}\right)\right] \omega^{\prime}\left(g^{\prime}\right)\left|\operatorname{det} g^{\prime}\right|(1-m) / 2 d v d g^{\prime} \\
=\int f^{\prime}\left(g^{\prime}\right) \omega^{\prime}\left(g^{\prime}\right) d g^{\prime}=\hat{f}^{\prime}\left(\pi^{\prime}\right) .
\end{gathered}
$$

Thus, we find the right-hand side of $(22)$ is equal to $\hat{f}^{\prime}\left(\pi^{\prime}\right)$, as required.

\section{REFERENCES}

[B] J. Bernstein, P-invariant distributions on $\mathrm{GL}(N)$ and the classification of unitary representations of $G l(N)$ (non-archinedean case), in Lie Group Representations II, Springer Verlag Lecture Notes in Mathematics, vol. 1041, pp. 50-102.

[F] S. Friedberg, Poincaré series for GL $(n)$ : Fourier expansion, Kloosterman sums, and algebreo-geometric estimates, Math. Zeitschrift, 196 (1987), 165188.

[G] D. Goldfeld, Kloosterman zeta functions for $\mathrm{GL}(n, \mathbf{Z})$, Proc. Intern. Cong. Math., Berkeley, 1 (1986), 417-424.

[H-R] M. Heumos and S. Rallis, Symplectic-Whittaker models for GL $(n)$, to appear in Pacific J. Math.

[H-L-R] G. Harder, R. R. Langlands and M. Rapoport, Algebraische Zyklen auf Hilbert-Blumenthal Flächen, J. für Math., 366, 53-120.

[J] H. Jacquet, On the nonvanishing of some L-functions, Proc. Indian Acad. Sci. (Math. Sci.), 97 (1987), 117-155.

[J-R] H. Jacquet and S. Rallis, Symplectic periods, to appear in J. Reine Angew. Math.

[J-Y] $\quad \mathrm{H}$. Jacquet and Y. Ye, Une remarque sur le changement de base quadratique, to appear in the Comptes Rendus de l'Académie des Sciences.

[K] A. A. Klyachko, Models for the complex representations of the groups $\mathrm{GL}(n, g)$, Math. USSR Sbornik, 48 (1984).

[M-W] C. Meglin and J. L. Waldspurger, Le spectre résiduel de $\mathrm{GL}(n)$, Ann. Scien. Ec. Norm. Sup., 22 (1989), 605-674.

[S] T. A. Springer, Some results on algebraic groups with involutions, in Algebraic Groups and Related Topics, Advanced Studies in Math., 6, Tokyo (1984), 323-343. 
[W I] J. L. Waldspurger, Correspondence de Shimura, J. Math. Pure Appl., 59 (1980), 1-113.

[W II] _ _ Sur les coefficients de Fourier des formes modulaires de poids demientier, J. Math. Pure Appl., 60 (1981), 375-484.

[Y] Y. Ye, Kloosterman integrals and base change for GL(2), J. Reine Angew. Math., 400 (1989), 57-121.

Received February 11, 1991 and in revised form May 1, 1991. The first author was partially supported by NSF grant DMS-88-01759. The second author was partially supported by NSF grant DMS-87-04375.

Columbia University

NEW YoRK, NY 10027

AND

The OHIo State University

Columbus, OH 43210 



\section{PACIFIC JOURNAL OF MATHEMATICS EDITORS}

\author{
V. S. VARADARAJAN \\ (Managing Editor) \\ University of California \\ Los Angeles, CA 90024-1555 \\ Herbert Clemens \\ University of Utah \\ Salt Lake City, UT 84112 \\ F. Michael Christ \\ University of California \\ Los Angeles, CA 90024-1555 \\ THOMAS ENRIGHT \\ University of California, San Diego \\ La Jolla, CA 92093
}

\author{
Nicholas ERcolani \\ University of Arizona \\ Tucson, AZ 85721 \\ R. FINN \\ Stanford University \\ Stanford, CA 94305 \\ VAughan F. R. JONES \\ University of California \\ Berkeley, CA 94720 \\ C. C. Moore \\ University of California \\ Berkeley, CA 94720 \\ MARTIN ScharlemanN \\ University of California \\ Santa Barbara, CA 93106 \\ Harold Stark \\ University of California, San Diego \\ La Jolla, CA 92093
}

STEVEN KeRCKHOFF

Stanford University

Stanford, CA 94305

\begin{tabular}{|c|c|c|c|c|}
\hline \multicolumn{5}{|c|}{ ASSOCIATE EDITORS } \\
\hline R. Arens & $\begin{array}{ll}\text { E. F. BECKENBACH } & \text { B. H } \\
(1906-1982) & \end{array}$ & NeUmanN & $\begin{array}{c}\text { F. WoLF } \\
(1904-1989)\end{array}$ & K. Yoshida \\
\hline \multicolumn{5}{|c|}{ SUPPORTING INSTITUTIONS } \\
\hline \multicolumn{2}{|c|}{ UNIVERSITY OF ARIZONA } & \multicolumn{3}{|c|}{ UNIVERSITY OF OREGON } \\
\hline \multicolumn{2}{|c|}{ UNIVERSITY OF BRITISH COLUMBIA } & \multicolumn{3}{|c|}{ UNIVERSITY OF SOUTHERN CALIFORNIA } \\
\hline \multicolumn{2}{|c|}{ CALIFORNIA INSTITUTE OF TECHNOLOGY } & \multicolumn{3}{|c|}{ STANFORD UNIVERSITY } \\
\hline \multicolumn{2}{|c|}{ UNIVERSITY OF CALIFORNIA } & \multicolumn{3}{|c|}{ UNIVERSITY OF HAWAII } \\
\hline \multicolumn{2}{|c|}{ MONTANA STATE UNIVERSITY } & \multicolumn{3}{|c|}{ UNIVERSITY OF TOKYO } \\
\hline \multicolumn{2}{|c|}{ UNIVERSITY OF NEVADA, RENO } & \multicolumn{3}{|c|}{ UNIVERSITY OF UTAH } \\
\hline & \multicolumn{3}{|c|}{ WASHINGTON STATE UNIVERSITY } \\
\hline \multicolumn{2}{|c|}{ OREGON STATE UNIVERSITY } & \multicolumn{3}{|c|}{ UNIVERSITY OF WASHINGTON } \\
\hline
\end{tabular}

The Supporting Institutions listed above contribute to the cost of publication of this Journal, but they are not owners or publishers and have no responsibility for its content or policies.

Mathematical papers intended for publication in the Pacific Journal of Mathematics should be in typed
form or offset-reproduced (not dittoed), double spaced with large margins. Please do not use built up fractions
in the text of the manuscript. However, you may use them in the displayed equations. Underline Greek letters
in red, German in green, and script in blue. The first paragraph must be capable of being used separately as
a synopsis of the entire paper. In particular it should contain no bibliographic references. Please propose a
heading for the odd numbered pages of less than 35 characters. Manuscripts, in triplicate, may be sent to any
one of the editors. Please classify according to the 1991 Mathematics Subject Classification scheme which
can be found in the December index volumes of Mathematical Reviews. Supply name and address of author
to whom proofs should be sent. All other communications should be addressed to the managing editor, or
Elaine Barth, University of California, Los Angeles, California $90024-1555-05$.
There are page-charges associated with articles appearing in the Pacific Journal of Mathematics. These
charges are expected to be paid by the author's University, Government Agency or Company. If the author or
authors do not have access to such Institutional support these charges are waived. Single authors will receive
50 free reprints; joint authors will receive a total of 100 free reprints. Additional copies may be obtained at
cost in multiples of 50 .

The Pacific Journal of Mathematics (ISSN 0030-8730) is published monthly except for July and August. Regular subscription rate: $\$ 190.00$ a year (10 issues). Special rate: $\$ 95.00$ a year to individual members of supporting institutions.

Subscriptions, orders for numbers issued in the last three calendar years, and changes of address should be sent to Pacific Journal of Mathematics, P.O. Box 969, Carmel Valley, CA 93924, U.S.A. Old back numbers obtainable from Kraus Periodicals Co., Route 100, Millwood, NY 10546.

The Pacific Journal of Mathematics at P.O. Box 969, Carmel Valley, CA 93924 (ISSN 0030-8730) is published monthly except for July and August. Second-class postage paid at Carmel Valley, California 93924, and additional mailing offices. Postmaster: send address changes to Pacific Journal of Mathematics, P.O. Box 969, Carmel Valley, CA 93924.

PUBLISHED BY PACIFIC JOURNAL OF MATHEMATICS, A NON-PROFIT CORPORATION Copyright (C) 1992 by Pacific Journal of Mathematics 


\section{Pacific Journal of Mathematics}

\section{Vol. 154, No. $2 \quad$ June, 1992}

Manuel (Rodriguez) de León, J. A. Oubiña, P. R. Rodrigues and

Modesto R. Salgado, Almost $s$-tangent manifolds of higher order . . . . 201

Martin Engman, New spectral characterization theorems for $S^{2} \ldots \ldots \ldots 215$

Yuval Zvi Flicker, The adjoint representation $L$-function for GL $(n) \ldots \ldots 231$

Enrique Alberto Gonzalez-Velasco and Lee Kenneth Jones, On the range

of an unbounded partly atomic vector-valued measure . ............ 245

Takayuki Hibi, Face number inequalities for matroid complexes and

Cohen-Macaulay types of Stanley-Reisner rings of distributive

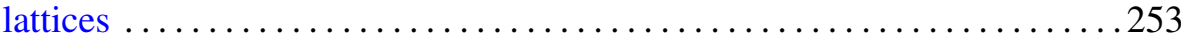

Hervé Jacquet and Stephen James Rallis, Kloosterman integrals for skew

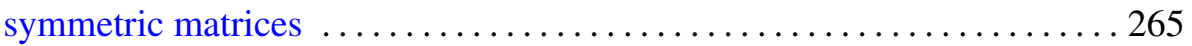

Shulim Kaliman, Two remarks on polynomials in two variables .........285

Kirk Lancaster, Qualitative behavior of solutions of elliptic free boundary problems ..........................................297

Feng Luo, Actions of finite groups on knot complements . ........... 317

James Joseph Madden and Charles Madison Stanton, One-dimensional

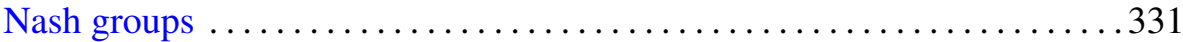

Christopher K. McCord, Estimating Nielsen numbers on

infrasolvmanifolds ......................................... 345

Gordan Savin, On the tensor product of theta representations of $\mathrm{GL}_{3}$ 369

Gerold Wagner, On means of distances on the surface of a sphere. II.

(Upper bounds) 